\title{
Effects of deprivation and presence of food on intake of sucrose solution in six-bottle tests'
}

DONALD H. OWINGS and ROBERT B. LOCKARD, University of Washington, Seattle, Wash.

Relative sucrose solution intake in 6-bottle tests was studied in rats under conditions of food-, water-, food-and-water-, and non-deprivation, and under conditions in which food was present or absent during testing. Water deprivation was most effective in altering the intake function, but deprivation interacted with the test-food variable to produce changes in the intake functions of food-, and food-and-water deprived Ss.

Different deprivation conditions have been found to have different effects upon intake of sugar solutions in rats. Food deprivation lowers the preference threshold for sucrose solutions (Campbell, 1958), but has little effect upon intake in a glucose vs water intake test (Jacobs, 1962). Water deprivation raises the sucrose preference threshold (Beck, Self, \& Carter, 1965), and increases water intake in glucose vs water intake tests (Jacobs, 1962). The effects of simultaneous food-and-water deprivation upon intake of glucose vs water have not been shown to differ from those of food deprivation alone (Jacobs, 1962).

The above cited studies have used only low concentrations of solution, or have given rats access to only two solutions. Failures to find differences in relative intake as a function of deprivation conditions could be attributed to insensitivity of the tests. Owings, Haerer, \& Lockard (1967) have used a six-bottle sucrose intake test with rats, a procedure which should be expected to provide a more sensitive test of the effects of deprivation, since the Ss are offered a wider variety of solutions to which to respond. Furthermore, Jacobs' (1962) study indicated that changes in relative intake occur within tests, a phenomenon to which a measure of total intake over several hours would be insensitive. One purpose of this study was, therefore, to compare the effects of food, water, food-and-water, and non-deprivation on sucrose intake in a six-bottle test, taking multiple within-test measures of intake.

The effects of presence or absence of dry food on sugar solution intake have not been systematically studied. During relatively long tests, Ss have been given ad lib access to food (Carpenter, 1958). When short tests were used, or when deprived Ss were compared with non-deprived Ss, food was not available during testing (Beck et al, 1965; Campbell, 1958). Jacobs (1962) found that the presence of food greatly enhanced water intake for rats deprived of only food, or of food and water in a glucose vs water intake test. A second objective of this study, then, was to examine the effects of presence or absence of food on relative sucrose solution intake under the four different deprivation conditions.

$$
\text { METHOD }
$$

The Ss were 64 male, Holtzman albino rats whose ages ranged from 90-120 days. Ss were maintained, four to a cage, in stainless steel cages ( $16 \times 10 \times 7$ in. high) with wire mesh floors and fronts. Water, when available, was presented in $1000 \mathrm{ml}$ polyethylene bottles, with stainless steel drinking tubes extending into the fronts of the cages. Food (Purina rat pellets), when available, was placed on cage floors. For testing, Ss were transferred to individual cages, identical to the maintenance cages. Test fluids were presented in six inverted $250 \mathrm{ml}$ polyethylene graduated cylinders, with stainless steel drinking tubes extending $1 / 4$ in. into the cage, 1-1/2 in. above the floor, and spaced $1-1 / 2$ in. apart. Positions of the various concentrations were randomized. Cage illumination during maintenance and testing was about $1.0 \mathrm{ft}-\mathrm{c}$.

Concentrations of sucrose used were 0 (distilled water), 2, 4, 8, 16 , and $32 \%$ by weight of solute and solvent; e.g., $4 \%$ is $4 \mathrm{~g}$ sugar in $96 \mathrm{~g}$ water (Pfaffman et al, 1954). All solutions were mixed with distilled water and were used only at room temperature. Fresh solutions were used for each test, and were allowed to stand $3 \mathrm{~h}$ before use.

The 64 Ss were divided into eight groups of eight rats each. Two groups were assigned to food deprivation, two to water deprivation, two to food-and-water deprivation, and two to non-deprivation. One group under each of the deprivation conditions was assigned to a food-present-during-testing condition, and one group to a food-absent-during-testing condition. The basic design, then, was a 4 by 2 factorial with independent groups.

All deprivation conditions were for $72 \mathrm{~h}$ duration. Testing was for a single $24 \mathrm{~h}$ period which began $3 \mathrm{~h}$ after onset of light in the $12: 12$ light-dark cycle. Readings of volume intake were taken at the end of each hour for the first $5 \mathrm{~h}$ and at the end of the $24 \mathrm{~h}$ period.

\section{RESULTS}

A deprivation by food-during-testing by concentration-of-testsolution by repeated-measures analysis of variance was performed upon the data (Analysis 1). The data were then broken down into six groups, each group consisting of the intake of a particular concentration under all treatment conditions. A deprivation by test-food by repeated-measures analysis of variance was performed upon each of these six groups of data (Analysis 2). Assumptions of homogeneity of covariance were violated in both analyses and the degrees of freedom for within-Ss effects and error terms were adjusted accordingly (Winer, 1962, p. 322).

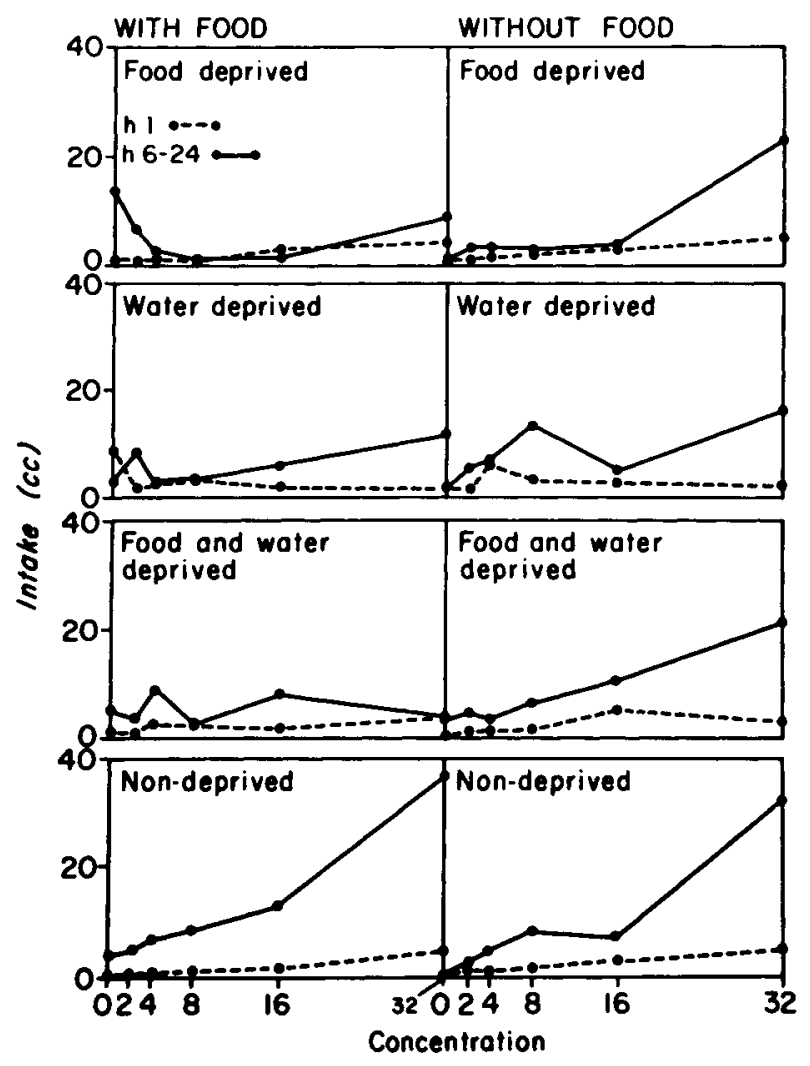

Fig. 1. Mean intake of sucrose solution as a function of concentration for all groups during Hour 1 and Hour 6-24. 
Mean intake of sucrose solution, during Hour 1 and Hours 6-24, is plotted as a function of concentration for each treatment condition in Fig. 1. In most cases intake was a monotonically increasing function of concentration, which is reflected in the highly significant concentration effect in Analysis $1(F=51.54$, $\mathrm{df}=1 / 56, \mathrm{p}<.01)$. There were, however, important deviations from the monotonically increasing function. Both water-deprived groups displayed highest intake of low concentrations during the first hour. The water-deprived group with food present during testing preferred wates, and the water-deprived group without food preferred $4 \%$ sucrose. Both groups exhibited shifts to preference for higher concentrations during subsequent test intervals. The food-deprived group with food present during testing exhibited very high intake of water during the last interval (Hours 6-24). This group had initially shown an intake function that increased monotonically with concentration. Behaving similarly to the food-deprived group, the food-and-water-deprived group with food present during testing exhibited high intake of $4 \%$ during the final interval (the high point at $16 \%$ is attributable to one S). This group, like the food-deprived group with food, had preferred higher concentrations during earlier intervals. These intra-test changes in preference as a function of test-food, and deprivation are reflected in significant deprivation by test-food by repeated-measures interactions at water $(F=4.78, \mathrm{df}=3 / 56$, $p<.01)$, and at $32 \%(F=4.78, d f=3 / 56, p<.01)$, and in $a$ near-significant interaction at $4 \%(\mathrm{~F}=2.02, \mathrm{df}=3 / 56$, $.1<\mathrm{p}<.25$ ) (Analysis 2 ).

\section{DISCUSSION}

Both ad lib groups exhibited monotonically increasing intake as a function of concentration for the whole $24 \mathrm{~h}$ period, replicating the results of Owings et al (1967) and demonstrating that the presence or absence of food has little effect on relative sucrose intake in non-deprived rats. On the other hand, although the food-, and food-and-water-deprived groups without food exhibited intake functions quite similar to those of the ad lib groups, the presence of food in these groups was found to be highly effective in changing the shapes of the intake functions. When food was present during testing, both groups displayed the monotonically increasing intake function during the early part of the test, but shifted to a preference for lower concentrations during the final period.

The effects of water deprivation were essentially in accord with other findings on the effects of this variable on sugar intake. The water-deprived groups' initial preference for low concentrations is in agreement with Jacobs' (1962) finding that water-deprived rats exhibited an initial preference for water over hypertonic glucose. It is possible, however, that Jacobs would have found a preference for hypotonic glucose over water had he used a wider range of solutions. The water-deprived group without food in this study drank more of $4 \%$ than of any other solution during the 1 st $h$. Also, Beck et al (1965) found that although thirsty rats displayed a higher preference threshold for sucrose, they nevertheless exhibited a preference for a hypotonic solution over water. The fact that hypotonic solutions are chosen over water suggests that, for thirsty rats, the osmotic dehydrating effects of hypertonic solutions mediate their rejection.

The water-deprived rats with food present apparently ate some of this food during the 1 st $h$, since they exhibited very high intake of water during this period. Just why these Ss did not "compromise" by drinking a hypotonic solution, as thirsty Ss without food did, is not clear. Ingestion of dry food could, through prior experience or innately, be associated with a "specific thirst" for water. Further evidence for this possibility can be found in the strong preference for water exhibited by the food-deprived rats with food during the final interval. Here again it is not clear why these Ss did not compromise by drinking a hypotonic solution, unless a specific thirst for water had been induced by the ingestion of dry food.

In conclusion, the test-food variable exercises its largest effect on extreme concentrations (water and $32 \%$ ), and makes a significant difference in relative sucrose intake only when rats are deprived and given food during testing, a procedure which no experimenter had previously followed. The deprivation variable itself makes a large difference only with water deprivation, but interacts strongly with the test-food variable primarily as a result of the effect of the presence of food on rats who are either food-deprived or both food-and-water-deprived.

\section{REFERENCES}

BECK, R. C., SELF, J. L., \& CARTER, D. J. Sucrose preference thresholds for satiated and water deprived rats. Psychological Reports, 1965, 16, 901-905.

CAMPBELL, B. A. Absolute and relative sucrose preference thresholds for hungry and satiated rats. Joumal of Comparative \& Physiological Psychology, 1958, 51, 795-800.

CARPENTER, J. A. A comparison of stimulus-presentation procedures in taste-preference experiments. Journal of Comparative \& Physiological Psychology, 1958, 51, 561-564.

JACOBS, H. L. Some physical, metabolic, and sensory components in the appetite for glucose. American Journal of Physiology, 1962, 203, 1043-1054.

OWINGS, D. H., HAERER, H. A., \& LOCKARD, R. B. Sucrose intake functions of rat and cockroach for single and six-solution presentations. Psychonomic Science, 1967, 7, 125.126.

PFAFFMAN, C., YOUNG, P. T., DETHIER, V. G., RICHTER, C. P., \& STELLAR, E. The preparation of solutions for research in chemoreception and food acceptance. Journal of Comparative \& Physiological Psychology, 1954, 47, 93-96.

WINER, B. J. Statistical principles in experimental design. New York: McGraw-Hill, 1962.

\section{NOTE}

1. This research was supported by Grant GB 3141 from the National Science Foundation. 